\title{
Cell Reprogramming: Mirage or Reality?
}

\section{Seyed Hadi Anjamrooz}

Cellular and Molecular Research Center, School of Medicine, Kurdistan University of Medical Sciences, Sanandaj, Iran

\begin{abstract}
Scientists should be in the service of science, not just in the service of humanity. Unfortunately, however, many researchers in the fields of regenerative medicine and cyto-therapy seem to have discriminated between scientific facts such as the efficiency and deficiency of reprogramming by concentrating their efforts mainly on the low efficiency of reprogramming while paying less attention to its high deficiency rate. This biased factualism has blurred some important aspects of cell behaviour. Additionally, any fascinating fact about cell behaviour may not actually be true and may be a mirage of the enchanting dance of the cell information content.
\end{abstract}

Keywords: Cell Reprogramming; Regenerative Medicine; Cell Behaviour; Cell

\section{Introduction}

In mammals, the zygote and early blastomeres are totipotent, whereas other cells possess less or no multi-lineage differentiation capacity [1]. It is thought that by better understanding the mystery underlying this cell behaviour, based on the architecture and kinetics of the cell information content, the promise of regenerative medicine can be fulfilled. However, instead of focusing on this, scientists have tried to find other ways to generate specialized cells. Eventually, their efforts led to new technologies that ostensibly enabled somatic cells to be reprogrammed into target cells. Currently, a majority of scientists believe that such reprogrammed cells are a promising source of cells for use in regenerative medicine. However, this hope may not be realized as expected because claims for both in vivo and in vitro observations of cell plasticity have remained highly controversial. These discrepancies may relate in part to cell-detection and cell-tracking strategies or differences in the sources of the original cells, cell purification techniques, or the approaches used to distinguish different cell-transformation and response processes [2-5]. Regardless of such contradictory evidence, the reprogramming process has been unsuccessful in many experimental instances that, because of the bias toward reporting "positive" results, either have been underreported or were reported but received less attention [5-15]. This failure is only half of the story. The other half is that even in cases of apparently successful reprogramming of cells, in addition to faulty reprogramming [16-21], the overall magnitude of reprogramming has been notoriously low [see Table S1 in the Supplemental Data available with this article], and some of the claims have proven difficult to reproduce in other laboratories, despite the use of similar or identical experimental paradigms $[6,7,22]$. Moreover, the published conclusions of some studies [15,23-25] have not been convincingly supported by the presented data [26], and because of potential errors, such as flaws in the experimental design or misinterpretations of data, much refinement and characterization of reprogrammed cells as well as their functionality and durability are necessary. For example, some tissues exhibit high levels of autofluorescence that can account for false positive results. This property, rather than the incorporation of donor cells, might explain the detection of fluorescent protein marker expression in recipient tissues [27]. Such auto-fluorescence can be particularly problematic when transdifferentiation of adult stem cells into non-autochthonous cell types is investigated in vivo. The fixation conditions and some auto-fluorophores, such as lipofuscin and flavin, may be responsible for the phenomenon of autofluorescence $[7,27,28]$. The production of lipofuscin appears to be symptomatic of membrane damage or damage to mitochondria and lysosomes, which are not unexpected in the damaged tissue of the recipient or during cell manipulation. Even if the experimental design is perfect, the temporary expression of a limited set of marker genes, as used in most studies, is often insufficient evidence from which to conclude that a cell has been permanently converted to a true stem cell or a new state of cellular differentiation. This behaviour, also known as "cellular mimicry," may be spontaneous or can arise from a cellular stress response [1,20,29-33]. The case in point is the activation of commonly used neural markers such as $\beta$-tubulin III, nestin, and NeuroD1 in skin or bone marrow-derived cells, which can reflect the cellular stress that occurs in response to removing cells from their particular microenvironments (or "niches") rather than demonstrating true trans-differentiation into the neural lineage [32,33]. In another case, it was revealed that myogenic conversion following the overexpression of the MyoD gene in muscle-unrelated cells is temporary $[20,30]$. Therefore, the dogma of irreversibility in cellular differentiation of terminally differentiated cells [34] appears to still be valid but not in as strict a form as previously thought. It is likely that cellular differentiation exhibits non-linear features of a bi-stable switch model of memory and mimicry, and either of these may predominate, depending on the external conditions [35]. Similarly, multipotent stem cells reversibly switch between states of dormancy and self-renewal [36]. Because quiescence has been postulated to protect stem cells from acquiring carcinogenic mutations, to hamper stem-cell exhaustion [36], and to increase cell resistance to anti-proliferative chemotherapeutic agents [37,38], it might make sense to postulate that the dormant state is the same state of temporary mimicry in which stem cells look like other cells. A similar dual property, known as meta-stability, has also been assumed for the pluripotent state [39]. Perhaps some of the contradictions and uncertainties in the literature related to the reliability of cell markers are attributable to the various cell-switch models noted above. For

*Corresponding author: Seyed Hadi Anjamrooz, Cellular and Molecular Research Center, School of Medicine, Kurdistan University of Medical Sciences, P.O. Box: 66177-13446, Sanandaj, Iran, Tel: +988716131382, Fax: +988716131383; E-mail: Hadi-Anjamrooz@muk.ac.ir

Received August 12, 2017; Accepted December 28, 2017; Published December 30, 2017

Citation: Anjamrooz SH (2017) Cell Reprogramming: Mirage or Reality? J Stem Cell Res Ther 7: 408. doi: 10.4172/2157-7633.1000408

Copyright: (c) 2017 Anjamrooz SH. This is an open-access article distributed unde the terms of the Creative Commons Attribution License, which permits unrestricted use, distribution, and reproduction in any medium, provided the original author and source are credited. 
instance, Lengner and colleagues (2007) reviewed numerous studies that had reported the critical role of Oct4 in somatic stem cell selfrenewal and pluripotency. By contrast, this research group, using tissuespecific ablation of Oct4, a transgenic Oct4-IRES-EGFP reporter, and quantitative RT-PCR, provided strong evidence suggesting that Oct4 expression is not required for somatic stem-cell function in several somatic tissues, including bone marrow (mesenchymal and hematopoietic lineages), intestinal epithelium, brain, liver, and hair follicles40. They argued that the Oct4 signals might be due to noise from the detection method, the presence of active Oct4 pseudogenes, or the expression of similar Pou-domain family members. In parallel, Liedtke et al. (2007) demonstrated that PCR detection of human Oct4 in cord blood and peripheral blood mononuclear cells is an artifact that is presumably caused by the amplification of Oct4 retropseudogenes or genomic DNA contamination [40,41]. However, unexpectedly, Oct4 expression was found to be absent in a number of pluripotent cells of the inner cell mass and was aberrantly expressed in trophoblast cells of the cloned blastocyst [19]. If so, and if cultivated cells can show temporary mimicry behaviors, then the use of Oct4 expression as a surrogate marker for induced pluripotent stem (iPS) cells is also of dubious value. Similarly, the actual nature of the donor cells in somatic cell nuclear transfer (SCNT) is still not completely clear. SCNT is the sole reprogramming technology that can produce cloned animals. To our knowledge, animal cloning by nuclear transfer has been applied to more than 20 different species; however, it remains markedly inefficient (Table S1). Claims for the generation of cloned animals using single-step nuclear transfer from terminally differentiated donor cells are not only based on poor and ambiguous evidence but are also redundant, given previous findings. Because, in the absence of stable markers that unambiguously identify fully differentiated cells, the use of a heterogeneous population of donor cells [9,42-44], and the greater amenability of less-differentiated cells to epigenetic reprogramming $[1,10,45]$ do not exclude the possibility that only rare stem cells present in the donor-cell samples could serve as donors for the very low percentage of viable clones, which is in contrast to what has been assumed previously [10,46-49]. Moreover, because only totipotent stem cells have the potential to give rise to all cells of an organism, including embryonic and extra-embryonic tissues [50], interestingly, it is claimed that a small percentage of adult or differentiated cells remain totipotent $[9,44]$. Hence, additional testing is required to confirm or discredit the previous ambiguous results. In this regard, several research groups have claimed success in producing monoclonal mice from mature immune cells [23] and from post-mitotic neurons [15,24] using a two-step cloning approach. In this procedure, ES cell lines are derived from cloned blastocysts, and the cloned mice are then generated from the EScell lines via tetraploid complementation. Although their results unequivocally demonstrated that highly differentiated cells can be reprogrammed, because the number of live offspring was quite low ( $0.001 \%)$ [51], the possible involvement of stem cells as donors for the generation of cloned blastocysts cannot be completely ruled out. Regardless of this possibility, it is unlikely that correct reprogramming occurred in these cases. First, using ES-cell intermediates in the second step may have allowed time for extra reprogramming [51]. Second, because placental problems are one of the most consistent defects in cloned mammals [24,52,53] the existence of correct nuclear reprogramming in placental tissues was not assessed.

\section{Many Steps in An Erroneous Direction}

Further modifications to cell reprogramming techniques and manipulations of culture conditions may not be able to significantly improve the applicability of reprogramming approaches in regenerative medicine. Although it seems unlikely, in vitro systems that are similar to real tissue environments are the best that we can hope to develop. However, the deficiency and inefficiency of reprogramming strategies are fundamentally different problems than achieving an optimal reprogramming system. This is because the current methods of pluripotent reprogramming, including the formation of iPS cells and SCNT, are not applicable for in vivo regeneration. Additionally, although lineage reprogramming, which includes dedifferentiation and transd-ifferentiation, can apparently be achieved in vivo, it carries an oncogenic risk [50], and its efficiency is very low (Table S1) and even inefficient in many cases. For example, purified hematopoietic stem cells (HSCs) failed to generate cardiomyocytes when directly transplanted into the myocardium $[5,11]$. Similarly, engrafted bone marrow cells did not transdifferentiate into neural cells [12], and intravenously infused neural stem cells did not contribute to hematopoiesis6. Moreover, in chimeric animals generated by the transplantation of a single green fluorescent protein-marked HSC, it has been rigorously shown that transdifferentiation of circulating HSCs into non-hematopoietic cells, including those of brain, kidney, gut, liver, and muscle, is an extremely rare event, if it occurs at all [7]. Even the physiological differentiation of transplanted adult stem cells occurred with very low efficiency in allogeneic recipients [54]. Neither exogenous nor endogenous cells could significantly reprogram to other lineages. An example of a recent finding in support of this notion is the direct conversion of pancreatic exocrine cells into endocrine $B$ cells in vivo using the bHLH transcription factor Neurogenin 3 in combination with Mafa and Pdx1 [55]. Nevertheless, in this case, a significant number of the transfected exocrine cells did not switch to insulin-producing $\beta$ cells. In another in vivo study, Qian et al. claimed to have performed virus-based cardiac reprogramming; however, the reprogramming occurred at a low frequency, similar to that observed in vitro Nygren et al. [56] used highly sensitive methods to detect low levels of mobilized hematopoietic stem and progenitor cells in mice that had experienced myocardial infarction. Intriguingly, they did not observe any increase in peripheral blood cellularity or progenitor/ stem cell activity. They also found no evidence for the trapping of potentially mobilized cells in the damaged myocardium or spleen of the infarcted mice [57]. Therefore, considering these studies and the very low efficiency of the different reprogramming techniques and their dire consequences, it seems unwise to apply these strategies clinically. Perhaps it is due to shortcomings such as these that scientists have so far failed to translate many of the claimed laboratory advances into clinically useful therapies. However, many companies and clinics throughout the world, such as in the United States, Japan, China, Russia, and Thailand, have injected paying customers with unproven preparations, regardless of the fact that the US Food and Drug Administration has not approved any such cell treatment for routine clinical use [58,59]. It will be very interesting to identify the main hurdle that is holding legal, ethical and efficient clinical cell therapy back. As an answer to this question, the author has developed the cell memory disc (CMD) theory [60-62]. Based on the new version, which is also called the 'every cell theory', this issue may be closely related to CMD fluidity.

\section{CMD Fluidity}

Theoretically, the basic unit of body structure consists of two stereotypical and radical pictures, the cell and the CMD, respectively. They are two dissimilar faces of the same coin. Every CMD in an organism gradually develops from various memorized layers of genetic, epigenetic, and non-genetic information [see Glossary] [60]. The information carried by the CMD has a unique architecture at any given moment, a feature that is called CMD fluidity. A biological cell, with the help of CMD fluidity, is able to exhibit different morphologies that 
may or may not be detectable by routine methods. The cell's detectable morphologies are represented by the forms of cell diversity and heterogeneity, while the undetectable forms seem to be similar. As every cell theory supposes the atom to be the building block of information, quantum mechanics may help to explain the fluid nature of the CMD, at least through the total atomic spin of the cell's atomic structure, which is not constant over time and space and determines all cell behaviours. It is possible that the cell's bistability and metastability $[35,39]$ are two reflections by which the CMD expresses its fluid nature. On the other hand, considering entropy and chirality [see Glossary], even in a population of apparently similar cells that express a particular marker, their CMDs may be arranged differently on the atomic and molecular levels. This means that attempts to apply one or even a limited set of cellular signatures are misguided and that there may be no completely reliable and specific cell marker.

Niche-specific signals can contribute to CMD fluidity, a property that is usually undetectable in the primary niche but detectable when the cell moves to other niches, either in vitro or in vivo. Cell isolation and cultivation can easily place a cell into a new niche, and similar to the scenario in invertebrates [63], cell migration from one in vivo niche to another is also likely. Metaphorically, the CMD's layers and the niche are similar to a piano's keys and musical notation, respectively. This is also reminiscent of paintings of doors by Georgia O'Keeffe [see Glossary]. Based on CMD fluidity, in addition to similar cells in different tissues, a population of a particular cell type within a given tissue or in a cell suspension may represent a spectrum of cells with heterogeneous CMDs. This important theoretical notion is in accordance with previous reports describing the heterogeneity of differentiated cells. Neuronal diversity in the olfactory sensory epithelium [24] serves as an example in this regard. It also appears that, due to cell heterogeneity resulting from the cell's information fluidity, chromatin changes associated with reprogramming do not occur homogenously in all cells transduced with reprogramming factors, and therefore, the reprogramming of individual differentiated cells within a given population seems to be completely random [50]. The highly significant differences between the gene expression profiles of several lineage-specific stem cells $[64,65]$ and the different capabilities of stem and progenitor cells within a tissue or in culture $[32,36,66]$ suggest that cell heterogeneity is not merely limited to the CMDs of differentiated cells. CMD heterogeneity can be observed even within highly blank CMDs. In support of this assertion, it has been demonstrated that, in addition to the distinct self-renewal probabilities and differing developmental potentials, the expression levels of key factors, such as Nanog, are highly variable in embryonic stem cells (ESCs) [67,68]. CMD fluidity highlights the difference between the CMDs of human cells and those of equivalent cells from laboratory animals that makes it difficult to extrapolate lab animal results to clinical practice. For example, it has been shown that human ESCs are phenotypically and functionally distinct from mouse ESCs [69-72].

\section{Conclusion and Perspectives}

In summary, based on CMD fluidity, some laboratory facts can be misleading. The future of regenerative therapy thus, in addition to unbiased factualism, depends on CMD-based regenerative strategies that are currently being developed. However, many details about the architecture and kinetics of the CMD during cell damage and repair must be deciphered if we expect to make significant progress in regenerative medicine.

\section{Supplemental Data}

Supplemental data include Table S1, which can be found with this article.

\section{Conflict of Interest Statement}

The author declares that he has no competing interests.

\section{References}

1. Jaenisch R, Young $R$ (2008) Stem cells, the molecular circuitry of pluripotency and nuclear reprogramming. Cell 132: 567-82 [PubMed].

2. Alvarez-Dolado M (2003) Fusion of bone-marrow-derived cells with Purkinje neurons, cardiomyocytes and hepatocytes. Nature 425: 968-73 [PubMed].

3. Beltrami AP (2003) Adult cardiac stem cells are multipotent and support myocardial regeneration. Cell 114, 763-776 [PubMed].

4. Zhang S (2004) Both cell fusion and transdifferentiation account for the transformation of human peripheral blood CD34-positive cells into cardiomyocytes in vivo. Circulation 110: 3803-3807 [PubMed].

5. Murry CE (2004) Haematopoietic stem cells do not transdifferentiate into cardiac myocytes in myocardial infarcts. Nature 428: 664-668 [PubMed].

6. Morshead CM, Benveniste P, Iscove NN, Van der Kooy D (2002) Hematopoietic competence is a rare property of neural stem cells that may depend on genetic and epigenetic alterations. Nat. Med. 8: 268-273 [PubMed]

7. Wagers A, Sherwood R, Christensen JL, Weissman IL (2002) Little evidence for developmental plasticity of adult hematopoietic stem cells. Science 297 2256-2259 [PubMed].

8. Wakayama T, Perry AC, Zuccotti M, Johnson KR, Yanagimachi R (1998) Fullterm development of mice from enucleated oocytes injected with cumulus cell nuclei. Nature 394: 369-374 [PubMed].

9. Gurdon JB, Byrne JA (2003) The first half-century of nuclear transplantation Proc. Natl. Acad. Sci. USA 100: 8048-8052 [PubMed].

10. Hochedlinger K, Jaenisch R (2002) Nuclear transplantation: lessons from frogs and mice. Curr. Opin. Cell. Biol. 14: 741-748.

11. Balsam LB (2004) Haematopoietic stem cells adopt mature haematopoietic fates in ischaemic myocardium. Nature 428: 668-673 [PubMed].

12. Castro RF (2002) Failure of bone marrow cells to transdifferentiate into neura cells in vivo. Science 297: 1299.

13. Choi JB (2003) Little evidence of transdifferentiation of bone marrow-derived cells into pancreatic beta cells. Diabetologia 46, 1366-1374 [PubMed].

14. Vallieres L, Sawchenko PE (2003) Bone marrow-derived cells that populate the adult mouse brain preserve their hematopoietic identity. J Neurosci 23 5197-207.

15. Li J, Ishii T, Feinstein P, Mombaerts P (2004) Odorant receptor gene choice is reset by nuclear transfer from mouse olfactory sensory neurons. Nature 428 393-399 [PubMed].

16. Polo JM (2010) Cell type of origin influences the molecular and functional properties of mouse induced pluripotent stem cells. Nat Biotechnol 28: 848-855.

17. Ng RK, Gurdon JB (2008) Epigenetic memory of an active gene state depends on histone $\mathrm{H} 3.3$ incorporation into chromatin in the absence of transcription. Nat. Cell. Biol. 10: 102-109 [PubMed]

18. Bortvin A (2003) Incomplete reactivation of Oct4-related genes in mouse embryos cloned from somatic nuclei. Development 130: 1673-1680.

19. Boiani M, Eckardt S, Schöler HR, McLaughlin KJ (2002) Oct4 distribution and level in mouse clones: consequences for pluripotency. Genes Dev. 16: 12091219 [PubMed].

20. Gurdon JB, Melton DA (2008) Nuclear reprogramming in cells. Science 322 1811-1815.

21. Kim K (2010) Epigenetic memory in induced pluripotent stem cells. Nature 467 285-290.

22. Massengale M, Wagers AJ, Vogel H, Weissman IL (2005) Hematopoietic cells maintain hematopoietic fates upon entering the brain. J Exp Med 201:1579-1589[PubMed]. 
23. Hochedlinger $K$, Jaenisch $R$ (2002) Monoclonal mice generated by nuclear transfer from mature B and T donor cells. Nature 415: 1035-1038.

24. Eggan K (2004) Mice cloned from olfactory sensory neurons. Nature 428: 44-49 [PubMed].

25. Sung LY (2006) Differentiated cells are more efficient than adult stem cells for cloning by somatic cell nuclear transfer. Nat Genet 38: 1323-1328.

26. Hochedlinger K, Jaenisch $R$ (2007) On the cloning of animals from terminally differentiated cells. Nat Genet 39: 136-137 [PubMed]

27. Jackson KA, Snyder DS, Goodell MA (2004) Skeletal muscle fiber-specific green autofluorescence: potential for stem cell engraftment artifacts. Stem Cells 22: 180-187.

28. Brazelton TR, Rossi FM, Keshet GI, Blau HM (2000) From marrow to brain: expression of neuronal phenotypes in adult mice. Science 290: 1775-1779 [PubMed].

29. Rizzino AA (2007) Challenge for regenerative medicine: proper genetic programming, not cellular mimicry. Dev Dyn 236: 3199-3207.

30. Weintraub H (1989) Activation of muscle-specific genes in pigment, nerve, fat, liver, and fibroblast cell lines by forced expression of MyoD. Proc Natl Acad Sci USA 86: 5434-5438 [PubMed].

31. Roobrouck VD, Vanuytsel K, Verfaillie CM (2011) Concise review: culture mediated changes in fate and/or potency of stem cells. Stem Cells. 29: 583-589.

32. Croft AP, Przyborski SA (2006) Formation of neurons by non-neural adult stem cells: potential mechanism implicates an artifact of growth in culture. Stem Cells. 24: 1841-1851 [PubMed].

33. Neuhuber B (2004) Reevaluation of in vitro differentiation protocols for bone marrow stromal cells: disruption of actin cytoskeleton induces rapid morphological changes and mimics neuronal phenotype. J. Neurosci Res 77: 192-204 [PubMed].

34. Stelling J, Sauer U, Szallasi Z, Doyle FJ, Doyle J (2004) Robustness of cellular functions. Cell 118: 675-85.

35. Wang L (2009) Bistable switches control memory and plasticity in cellular differentiation. Proc Natl Acad Sci USA 106: 6638-6643 [PubMed].

36. Wilson A (2008) Hematopoietic stem cells reversibly switch from dormancy to self-renewal during homeostasis and repair. Cell 135: 1118-1129.

37. Aguirre-Ghiso JA (2007) Models, mechanisms and clinical evidence for cancer dormancy. Nat Rev Cancer 7: 834-846 [PubMed].

38. Trumpp A, Wiestler OD (2008) Mechanisms of Disease: Cancer stem cellstargeting the evil twin. Nat. Clin Pract Oncol 5: 337-347.

39. Hanna J (2009) Metastable pluripotent states in NOD-mouse-derived ESCs. Cell Stem Cell 4: 513-24 [PubMed].

40. Lengner CJ (2007) Oct4 expression is not required for mouse somatic stem cell self-renewal. Cell Stem Cell 1: 403-415.

41. Liedtke S, Enczmann J, Waclawczyk S, Wernet P, Kögler G (2007) Oct4 and its pseudogenes confuse stem cell research. Cell Stem Cell 1: 364-366 [PubMed].

42. Lagasse E, Weissman IL (1996) Flow cytometric identification of murine neutrophils and monocytes. J. Immunol. Methods 197: 139-150.

43. Gurdon JB, Laskey RA, Reeves OR (1975) The developmental capacity of nuclei transplanted from keratinized skin cells of adult frogs. J Embryol Exp Morphol 34: 93-112 [PubMed].

44. Wilmut I, Schnieke AE, McWhir J, Kind A J, Campbell KH (1997) Viable offspring derived from fetal and adult mammalian cells. Nature 385: 810-813.

45. Li J, Greco V, Guasch G, Fuchs E, Mombaerts P (2007) Mice cloned from skin cells. Proc Natl Acad Sci USA 104: 2738-2743 [PubMed].
46. Rideout WM, Eggan K, Jaenisch R (2001) Nuclear cloning and epigenetic reprogramming of the genome. Science 293: 1093-1098.

47. Liu L (2001) Cloning efficiency and differentiation. Nat Biotechnol 19: 406-406 [PubMed].

48. Oback B, Wells D (2002) Donor cells for nuclear cloning: many are called, but few are chosen. Cloning Stem Cells 4: 147-168.

49. Weissman IL (2000) Stem cells: units of development, units of regeneration, and units in evolution. Cell 100: 157-168 [PubMed].

50. Jopling C, Boue S, Izpisua Belmonte C (2011) Dedifferentiation, transdifferentiation and reprogramming: three routes to regeneration. Nat Rev Mol Cell Biol 12: 79-89 (2011).

51. Rossant J (2002) A monoclonal mouse? Nature 415: 967-969.

52. Yang $X$ (2007) Nuclear reprogramming of cloned embryos and its implications for therapeutic cloning. Nat Genet 39: 295-302 [PubMed].

53. Eggan K (2001) Hybrid vigor, fetal overgrowth, and viability of mice derived by nuclear cloning and tetraploid embryo complementation. Proc. Natl Acad. Sci. USA 98: 6209-6214.

54. Jiang S (2004) Transplanted human bone marrow contributes to vascular endothelium. Proc Natl Acad. Sci USA 101: 16891-16896 [PubMed].

55. Zhou Q, Brown J, Kanarek A, Rajagopal J, Melton DA (2008) In vivo reprogramming of adult pancreatic exocrine cells to beta-cells. Nature 455 627-632.

56. Qian L (2012) In vivo reprogramming of murine cardiac fibroblasts into induced cardiomyocytes. Nature 485: 593-598.

57. Nygren JM (2004) Bone marrow-derived hematopoietic cells generate ardiomyocytes at a low frequency through cell fusion, but not transdifferentiation. Nat Med 10: 494-501[PubMed].

58. Cyranoski D (2010) Strange lesions after stem-cell therapy. Nature 465: 997

59. Cyranoski D (2012) Stem-cell therapy takes off in Texas. Nature 483: 13-14 [PubMed].

60. Anjamrooz SH (2013) The cellular memory disc of reprogrammed cells. Stem Cell Rev. 9: 190-209.

61. Anjamrooz SH (2015) Cell memory-based therapy. J Cell Mol Med. 11: 2682 2689 [PubMed].

62. Anjamrooz SH (2016) CMD kinetics and regenerative medicine. Am J Trans Res. 8: 1615-24

63. Xie T, Spradling AC (2000) A niche maintaining germ line stem cells in the Drosophila ovary. Science 290: 328-330 [PubMed]

64. Ivanova NB (2002) A stem cell molecular signature. Science 298: 601-604

65. Ramalho-Santos M, Yoon S, Matsuzaki Y, Mulligan RC, Melton DA (2002) "Stemness": transcriptional profiling of embryonic and adult stem cells. Science 298: 597-600 [PubMed].

66. Jessell TM (2000) Neuronal specification in the spinal cord: inductive signals and transcriptional codes. Nat Rev Genet 1: 20-29.

67. Silva J, Smith A (2008) Capturing pluripotency. Cell 132: 532-536 [PubMed].

68. Osafune K (2008) Marked differences in differentiation propensity among human embryonic stem cell lines Nat Biotechnol 26: 313-315.

69. Thomson JA (1998) Embryonic stem cell lines derived from human blastocysts Science 282: 1145-1147 [PubMed].

70. Boyer LA (2005) Core transcriptional regulatory circuitry in human embryonic stem cells. Cell 122: 947-956.

71. Silva SS, Rowntree RK, Mekhoubad S, Lee JT (2008) X-chromosome inactivation and epigenetic fluidity in human embryonic stem cells. Proc Natl Acad Sci USA 105: 4820-4825 [PubMed].

72. Tesar PJ (2007) New cell lines from mouse epiblast share defining features with human embryonic stem cells. Nature 448: 196-199. 